\title{
Antena impresa invertida reconfigurable en frecuencia a base de una microdescarga de plasma
}

\author{
Plasma based frequency tunable inverted microstrip antenna \\ Francisco Pizarro Torres ${ }^{1 *} \quad$ Romain Pascaud ${ }^{2} \quad$ Olivier Pascal $^{3}$ \\ Thierry Callegari ${ }^{3} \quad$ Laurent Liard $^{3}$
}

Recibido 8 de junio de 2015, aceptado 9 de mayo de 2016

Received: June 8, 2015 Accepted: May 9, 2016

\begin{abstract}
RESUMEN
En este artículo se propone el estudio de una antena impresa invertida reconfigurable en frecuencia a base de una microdescarga de plasma. La idea es generar una microdescarga controlada en corriente al interior de una cavidad situada entre el plano tierra de la antena y su conductor principal, cuyo objetivo final es de modificar la frecuencia de resonancia de la antena. El interés en la utilización del plasma como elemento reconfigurable es su tiempo de conmutación y, sobre todo, su capacidad de manejo de altas potencias. Se demostró de manera teórica que la reconfiguración en frecuencia de una antena tipo parche, con una microdescarga en su cavidad, es dependiente de la densidad electrónica del plasma. Además, se implementó un prototipo de antena impresa invertida reconfigurable en banda $\mathrm{X}$ integrando una microdescarga, la que tuvo excursión en frecuencia medida de $180 \mathrm{MHz}$.
\end{abstract}

Palabras clave: Antena reconfigurable, descarga de plasma, reconfiguración en frecuencia.

\begin{abstract}
This paper proposes the study of an inverted microstrip plasma-based reconfigurable antenna. The scope is to generate controlled microplasma inside the cavity between the antenna ground plane and its main conductor. This plasma will modify the resonant frequency of the antenna. The interest of using a plasma discharge as an active reconfigurable element is its switching time and mainly its capability of handling high microwave power. It was demonstrated, theoretically, that the reconfiguration frequency of a microstrip patch antenna with a microdischarge in its cavity, depends on the electron density of the plasma. Furthermore, a prototype of an X-Band inverted microstrip patch antenna, which integrates a microdischarge, was implemented. The measured frequency excursion for this prototype was $180 \mathrm{MHz}$.
\end{abstract}

Keywords: Reconfigurable antenna, plasma discharge, frequency tuning.

1 Escuela de Ingeniería Eléctrica. Pontificia Universidad Católica de Valparaíso. Av. Brasil 2147. Valparaíso, Chile. E-mail: francisco.pizarro.t@pucv.cl

2 ISAE-DEOS. Université de Toulouse. 10 Av Edouard Belin, BP 54032, 31055 Toulouse Cedex 4. Francia.

E-mail: romain.pascaud@isae-supaero.fr

3 CNRS, UPS, INP, LAPLACE. Université de Toulouse. 118, route de Narbonne 31062 Toulouse cedex 9. Francia.

E-mail: opascal@laplace.univ-tlse.fr; thierry.callegari@laplace.univ-tlse.fr; laurent.liard@laplace.univ-tlse.fr

* Autor de correspondencia 


\section{INTRODUCCIÓN}

Hoy en día, debido al aumento de los estándares de comunicación inalámbrica, las antenas reconfigurables en frecuencia han sido objeto de numerosos estudios [1]. Generalmente estas antenas integran elementos activos para realizar la reconfiguración en frecuencia (e.g. Diodos PIN, MEMS RF, varactores,...) o bien utilizan materiales reconfigurables (e.g. Ferritas, cristales líquidos, ...). Sin embargo, gran parte de esos elementos solo pueden ser utilizados en rangos bajos o medianos de potencia microondas. Es en este contexto donde el uso de las descargas de plasma como elemento para la reconfiguración que pueda manejar altas potencias de microondas ha sido estudiado [2-3].

El uso de descargas de plasma para la reconfiguración en antenas usualmente ha sido mediante el uso de grandes tubos de vidrio llenos con plasma, usados para modificar ya sea su frecuencia de resonancia o bien su patrón de radiación [4]. Sin embargo, estas soluciones son voluminosas respecto al dispositivo microondas. Una solución para este problema sería el uso de descargas de plasma de menor tamaño, conocidas como microdescarga, las que pueden ser compatibles con tecnologías de líneas y antenas impresas.

A pesar de los recientes avances en microdescargas [5], pocos estudios han sido realizados utilizando microdescarga integradas en dispositivos de tipo impreso [6]. En este artículo se presentará el estudio teórico y la realización de un prototipo de validación del concepto de una antena impresa reconfigurable en frecuencia a base de plasma.

\section{PLASMAS FRÍOS}

Un plasma es un gas ionizado, macroscópicamente neutro. Para generar una descarga de plasma, se debe entregar suficiente energía a un gas neutro para alterar su grado de ionización y el gas pase de un estado aislante hacia un estado conductor.

Una forma bastante utilizada para crear un plasma es mediante la aplicación de un voltaje entre dos electrodos en el gas. Si la tensión es suficiente se genera una descarga de plasma del tipo no-equilibrio o plasma frío entre los electrodos. Este plasma tiene como característica principal que sus partículas más pesadas (iones y neutros) tienen una temperatura cercana a la temperatura ambiente. Esta temperatura es mucho menor que la temperatura de los electrones (de ahí el término no-equilibrio), los que cuentan con la energía suficiente para realizar colisiones y mantener la ionización. Esta característica de temperatura hace posible su integración en circuitos de radiofrecuencia, específicamente en circuitos planares.

Un plasma frío homogéneo puede ser modelado como un medio dieléctrico complejo, cuya permitividad compleja puede ser escrita como:

$$
\begin{aligned}
& \epsilon_{p}=\epsilon_{r}(1-j \tan \delta) \\
& \epsilon_{p}=\left(1-\frac{\omega_{p}^{2}}{\omega^{2}+v_{p}^{2}}\right)\left(1-j \frac{v_{p}}{\omega} \frac{\omega_{p}^{2}}{\omega^{2}+v_{p}^{2}-\omega_{p}^{2}}\right)
\end{aligned}
$$

donde $\omega$ representa la frecuencia angular de la onda electromagnética que interactúa con el plasma ( $\mathrm{rad} / \mathrm{s})$, $v_{p}$ representa la transferencia de momento electrónneutro, conocida también como la frecuencia de colisión $(1 / \mathrm{s})$, y $\omega_{p}$ representa la pulsación plasma $(\mathrm{rad} / \mathrm{s})$, definida como:

$$
\omega_{p}=\sqrt{\frac{n_{e} q^{2}}{\epsilon_{0} m_{e}}}
$$

con $q$ la carga del electrón, $\varepsilon_{0}$ la permitividad del vacío, $m_{e}$ la masa del electrón y $n_{e}$ la densidad electrónica. Según las ecuaciones (1) y (2), se observa que la constante dieléctrica de un plasma frío homogéneo $\varepsilon_{r}$ depende de la densidad electrónica $n_{e}$ y de la frecuencia de colisión $v_{p}$. Estos parámetros a su vez dependen principalmente de parámetros externos controlables, como la potencia inyectada a la descarga (control sobre $n_{e}$ ) y del tipo de gas y su presión $\left(\right.$ control sobre $v_{p}$ ). Además podemos notar que según los valores que pueda tomar la frecuencia angular $\omega$ de la onda que interactúa con el plasma, la constante dieléctrica $\varepsilon_{r}$ puede tener valores negativos, nulos, o positivos entre 0 y 1 . Finalmente, una ventaja extra del plasma es que si no existe ionización, el gas se comporta como vacío.

\section{ANÁLISIS DEL MODELO DE CAVIDAD PARA UNA ANTENA IMPRESA RECTANGULAR CON PLASMA}

\section{Descripción del análisis del modelo de cavidad}

La técnica de análisis del modelo de cavidad es usada para estimar la frecuencia de resonancia de 
una antena impresa (microstrip) [7]. Esta consiste en modelar la antena como una cavidad cuyos límites superiores son condiciones de conductor eléctrico perfecto PEC (Perfect Electric Conductor), y su perímetro está condicionado con límites del tipo conductor magnético perfecto PMC (Perfect Magnetic Conductor) como lo muestra la Figura 1.

En este caso se considera la cavidad de largo $L$, ancho $W$ y altura $h$ llena completamente con un plasma frío homogéneo. En este modelo, la frecuencia angular de resonancia $\omega_{\text {res }}$ para el modo fundamental $\mathrm{TM}_{10}$ está dada por:

$$
\omega_{\text {res }}=\frac{c \pi}{L \sqrt{\epsilon_{r}}}=\frac{c \pi}{L \sqrt{1-\frac{\omega_{p}^{2}}{\omega^{2}+v_{p}^{2}}}}
$$

Si $\omega_{\text {res }} \gg>\omega_{p}$ y $\omega_{\text {res }} \gg>v_{p}$ podemos asumir que la permitividad del plasma es positiva (i.e. $0<\varepsilon_{r}$ $<1$ ), obtenemos entonces la siguiente función bicuadrática para $\omega_{\text {res }}$ :

$$
\omega_{r e s}^{4}+\left(v_{p}^{2}-\omega_{p}^{2}-\frac{c^{2} \pi^{2}}{L^{2}}\right) \omega_{r e s-}^{2}-\left(\frac{c \pi v_{p}}{L}\right)^{2}=0
$$

La ecuación (4) que tiene cuatro soluciones, de las que solo una de ella es real y positiva. Esta solución representa entonces la frecuencia de resonancia angular de la cavidad $\omega_{\text {res }}$ cuando esta se encuentra llena de un medio de plasma.

\section{Resultados del análisis del modelo de cavidad}

Para este estudio de modelo de cavidad consideraremos una microdescarga de plasma generada en una mezcla

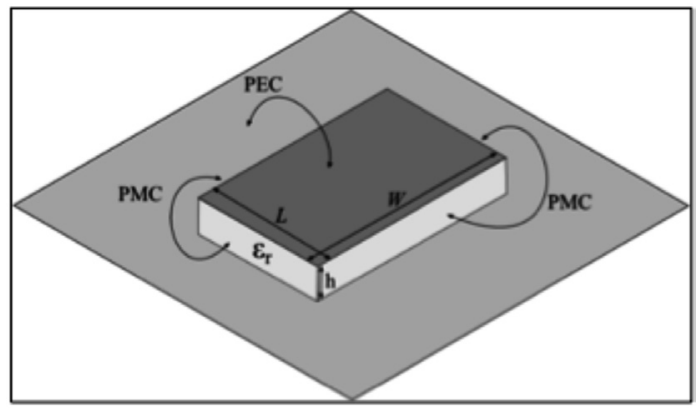

Figura 1. Modelo de cavidad para el cálculo de la frecuencia de resonancia en antenas impresas. de Neón-Xenón a una presión de 5 torr (1 atm $=760$ torr). Para este tipo de gas, a esa presión, tenemos una frecuencia de colisión aproximada de $v_{p}=6,5 \times 10^{9} \mathrm{~s}^{-1} \mathrm{y}$ una densidad electrónica máxima de $n_{e}=3 \times 10^{11} \mathrm{~cm}^{-3}[5]$.

La Figura 2 muestra la frecuencia de resonancia $f_{\text {res }}$ calculada gracias a la ecuación (4) y la tangente de pérdidas dieléctricas $\tan _{\delta}$ en función de la densidad electrónica $n_{e}$. Se observa que la frecuencia de resonancia de la cavidad aumenta casi linealmente junto con la densidad electrónica. A partir de esta condición, uno puede teóricamente controlar la frecuencia de resonancia de la cavidad variando la densidad electrónica del plasma (e.g. aumentando la potencia inyectada a la descarga) [8].

Otro punto a notar en la Figura 2 es que un aumento de la densidad electrónica en la cavidad implica un aumento en la tangente de pérdidas dieléctricas $\tan \delta$ del plasma.

\section{MODELO Y RESULTADO DE SIMULACIÓN}

\section{Antena impresa invertida cargada con una microdescarga}

Numerosas simulaciones han sido realizadas con Ansoft HFSS. La Figura 3 muestra un diagrama de la antena simulada. Esta consiste en una antena impresa invertida la que contiene una microdescarga de plasma entre su plano tierra y su conductor principal. La antena invertida es una antena rectangular impresa sobre un sustrato con $\varepsilon_{r}=3,5$ y $\tan _{\delta}=0,0018$. Sus dimensiones laterales son

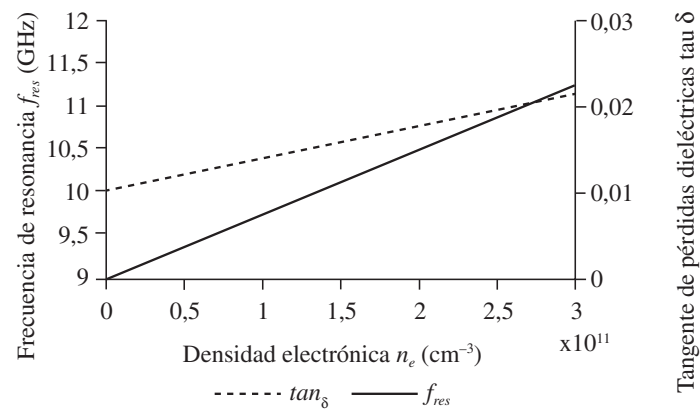

Figura 2. Frecuencia de resonancia de la cavidad (modo $\mathrm{TM}_{10}$ ) y su correspondiente tangente de pérdidas dieléctricas en función de la densidad electrónica $n_{e}[8]$. 
$L=11,35 \mathrm{~mm}$ y $W=18 \mathrm{~mm}$, y está adaptada para una frecuencia de resonancia de $10 \mathrm{GHz}$.

La antena es alimentada por una línea de transmisión impresa invertida de $50 \Omega\left(\mathrm{W}_{\text {linea }}=3,3 \mathrm{~mm}\right)$. Debido a esta configuración invertida, existe un espacio de aire de $1 \mathrm{~mm}$ entre el plano tierra de la antena y el sustrato dieléctrico en el que está impresa la antena. Es en este espacio donde la descarga de plasma será alojada. La descarga simulada tiene los mismos valores de largo y ancho de la antena $(L$ y $W)$, a diferencia de su altura, que es ligeramente inferior a la altura de la cavidad ( $h_{\text {plasma }}=0,8 \mathrm{~mm}$ ). Esta consideración en la simulación es para acercarse al fenómeno de las envolturas que poseen estas descargas de plasma que aparecen entre los dos conductores de la antena [5].

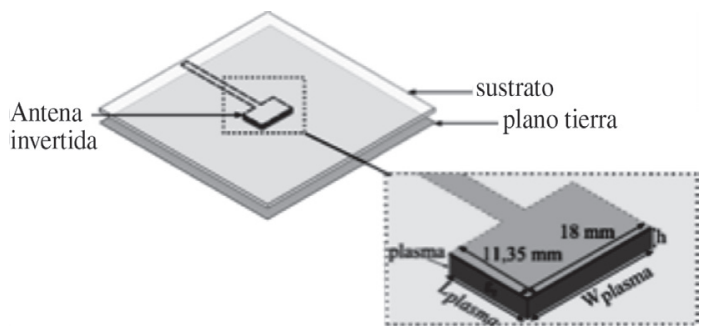

Figura 3. Antena impresa invertida simulada con descarga de plasma.

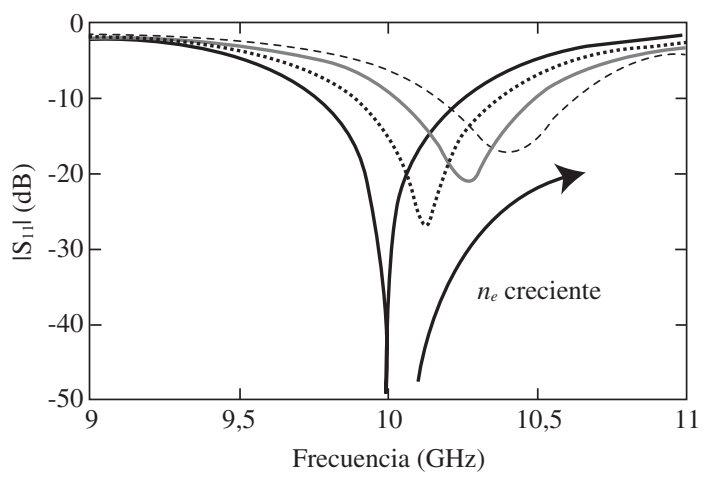

- Sin plasma $\cdots \cdots \cdot n_{e}=1 \times 10^{11} \mathrm{~cm}^{-3}$
$-\cdots n_{e}=2 \times 10^{11} \mathrm{~cm}^{-3} \quad n_{e}=3 \times 10^{11} \mathrm{~cm}^{-3}$

Figura 4. Parámetro $\left|S_{11}\right|$ de la antena impresa invertida con una microdescarga de plasma en función de la frecuencia para distintas densidades electrónicas $n_{e}\left(v_{p}\right.$ $\left.=6,5 \times 10^{9} \mathrm{~s}^{-1}\right)[8]$.

\section{Resultados de simulación de la antena invertida con microdescarga}

La Figura 4 presenta los resultados simulados del parámetro $\left|\mathrm{S}_{11}\right|$ de la antena invertida impresa con la descarga de plasma, en función de la frecuencia y de la densidad electrónica del plasma $\left(v_{p}=6,5\right.$ $\times 10^{9} \mathrm{~s}^{-1}$ ).

Notamos que la frecuencia de resonancia de la antena aumenta junto con el incremento de la densidad electrónica del plasma. Es observado un aumento de $400 \mathrm{MHz}$ (i.e. $4 \%$ de la frecuencia de resonancia inicial) cuando $n_{e}=3 \times 10^{11} \mathrm{~cm}^{-3}$. Este aumento es menor al predicho anteriormente con el análisis del modelo de cavidad. Esta diferencia se puede explicar ya que la configuración de antena invertida no tiene las mismas condiciones de configuración que una antena impresa clásica utilizada para el modelo de cavidad.

\section{PROTOTIPO Y RESULTADO EXPERIMENTAL}

\section{Prototipo de antena impresa invertida}

Un prototipo de antena invertida impresa ha sido diseñado y medido. En la Figura 5 se muestra la antena impresa. Esta antena está impresa en un sustrato TACONIC RF-35 de 1,52 mm de espesor, con una tangente de pérdidas $\tan _{\delta}=0,0018$ y una constante dieléctrica $\varepsilon_{r}=3,5$. Las dimensiones de la antena son las mismas que la simulada con Ansoft HFSS $(L=11,35 \mathrm{~mm}$ y $W=18 \mathrm{~mm})$. Podemos ver sobre la antena un circuito de alimentación DC sobre la línea de alimentación RF para poder ocupar el conductor de la antena como ánodo y generar la descarga.

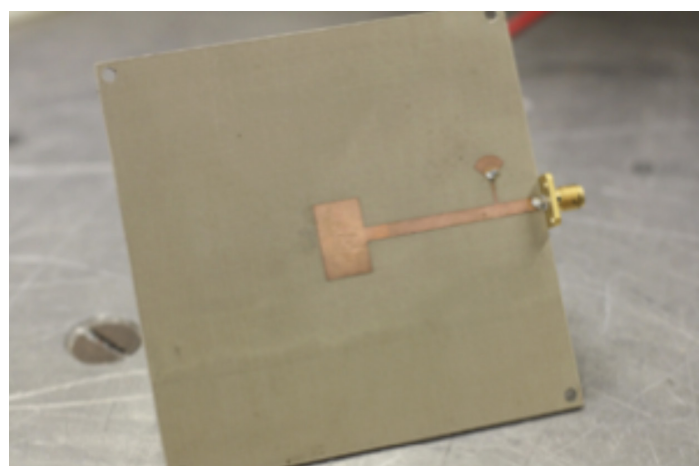

Figura 5. Antena invertida impresa sobre sustrato. 
La Figura 6 muestra un esquema de despiece del prototipo. Se pueden apreciar las piezas que componen la estructura de antena inversa impresa con el circuito que permite generar la descarga de plasma (cátodo y ánodo). En la Figura 7 se muestra el prototipo montado sobre su estructura final en PVC, la que sostiene toda la estructura RF.

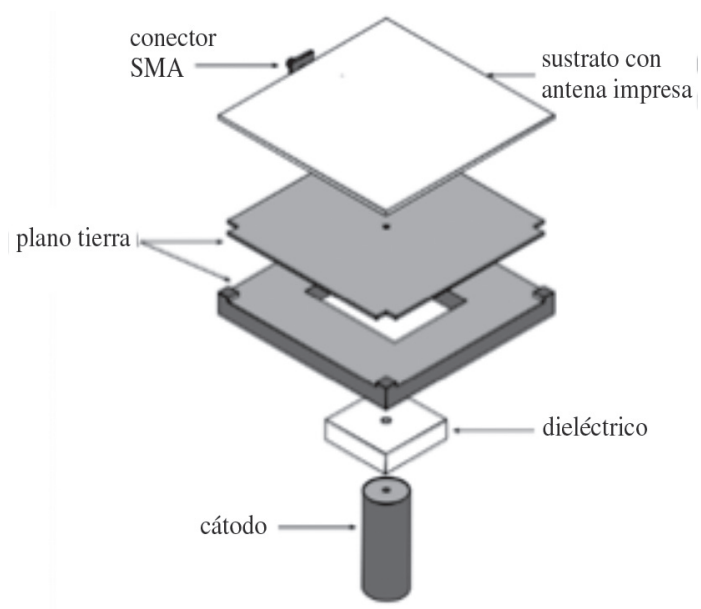

Figura 6. Esquema de despiece de la antena impresa invertida con estructura generadora de plasma.

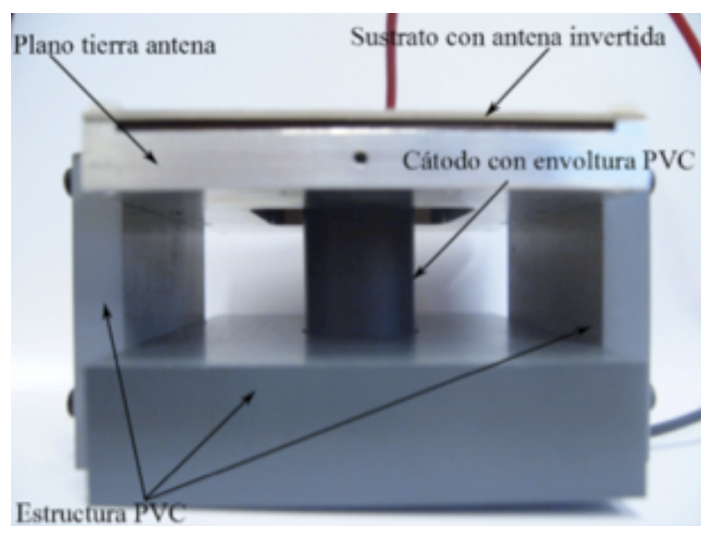

Figura 7. Antena invertida impresa con estructura generadora de microdescarga, montada sobre su estructura en PVC.

La Figura 8 muestra el parámetro $\left|S_{11}\right|$ de la antena simulada y la antena medida sin plasma con un analizador vectorial (VNA). Vemos que la frecuencia de resonancia medida es de $10,63 \mathrm{GHz}$, a diferencia de los $10 \mathrm{GHz}$ de la misma antena diseñada en simulación. Esto se puede explicar a que la distancia entre el conductor principal de la antena y su plano tierra no es exactamente de $1 \mathrm{~mm}$ (tolerancia de fabricación), lo que aumentaría la frecuencia de resonancia de la antena si esta distancia es menor a la simulada [9].

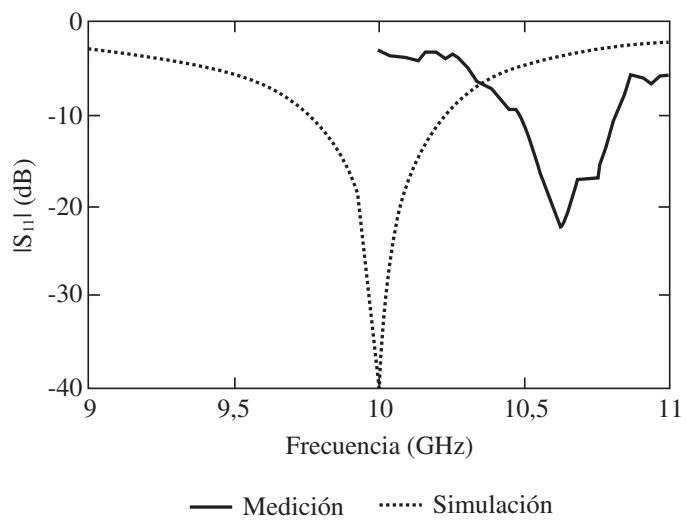

Figura 8. Parámetro $\left|S_{11}\right|$ de la antena impresa invertida simulada y la misma antena medida sin plasma.

\section{Protocolo experimental}

El protocolo experimental se muestra en la Figura 9. El dispositivo RF es insertado en una cámara de vacío acondicionada con conectores especiales que permiten la conexión de diversos circuitos de control. Uno de los circuitos corresponde a la unidad de control de la presión y del tipo de gas, y una segunda sección de control de la corriente DC inyectada a la descarga. Para medir el parámetro $\left|S_{11}\right|$, la antena se conecta a un VNA.

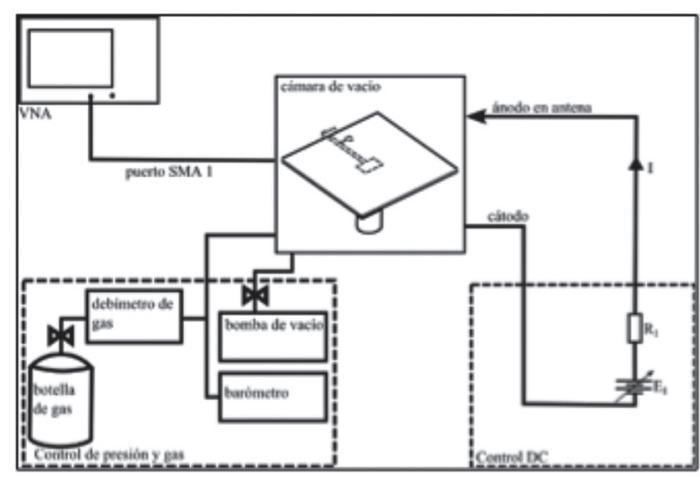

Figura 9. Protocolo experimental. 
El tipo de gas utilizado para las mediciones será una mezcla de Neón-Xenón (99\% Neón, 1\% Xenón) a una presión de 5 torr $\left(v_{p}=6,5 \times 10^{9} \mathrm{~s}^{-1}\right)$. La corriente inyectada a la descarga varía de 0 a $100 \mathrm{~mA}$. Es la variación de esta corriente la que permite una variación de la densidad electrónica.

\section{Resultado de mediciones}

La Figura 10 muestra el parámetro $\left|S_{11}\right|$ medido de la antena impresa invertida en función de la frecuencia y de la corriente inyectada a la descarga.

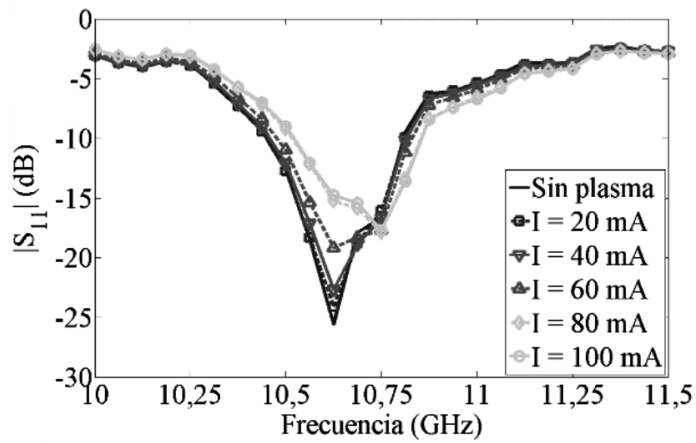

Figura 10. Parámetro $\left|\mathrm{S}_{11}\right|$ medido de la antena impresa invertida en función de la frecuencia y de la corriente inyectada a la descarga.

Tal como fue predicho con el modelo de cavidad, la frecuencia de resonancia de la antena aumenta junto con el aumento de la corriente (por ende de la densidad electrónica). El máximo de desplazamiento obtenido es de $180 \mathrm{MHz}$ cuando se inyecta una corriente de $100 \mathrm{~mA}$ a la descarga. Este aumento es menor que el simulado (400 MHz). Esto se puede explicar debido a la forma que tomó la descarga en el dispositivo. Se pudo ver en el dispositivo experimental, que el plasma no ocupó en su totalidad la cavidad entre el plano tierra de la antena y su conductor principal, lo que acarrea como consecuencia una disminución de la interacción de la onda RF y el plasma.

\section{CONCLUSIONES}

Se ha demostrado de manera teórica, en simulación y de forma experimental, la realización de una antena impresa invertida que utiliza una microdescarga como elemento activo para la reconfiguración en frecuencia.
Se utilizó el modelo de cavidad para antenas impresas con el fin de demostrar el principio de reconfiguración en frecuencia en función de la densidad electrónica del plasma y luego se simuló una antena impresa invertida con una descarga de plasma. Se observó que en ambos casos hubo un aumento de la frecuencia de resonancia en función de la densidad electrónica del plasma.

Finalmente se procedió a una demostración experimental del principio de reconfiguración en frecuencia. Se obtuvo un desplazamiento máximo de $180 \mathrm{MHz}$ de la frecuencia de resonancia. Si bien este desplazamiento en frecuencia es menor que el esperado en simulación, se debe a que la descarga no ocupó toda la cavidad, disminuyendo así la interacción entre el plasma y la onda RF.

Como perspectiva, se está trabajando en una topología de generación de descarga que permita llenar la cavidad, y así poder aumentar la interacción entre el plasma y la onda RF. Otra perspectiva es de reducir, en lo posible, las pérdidas ocasionadas por el plasma.

\section{AGRADECIMIENTOS}

Los autores agradecen a PRES y la región MidiPyrénées, Francia, por su financiamiento en este proyecto.

\section{REFERENCIAS}

[1] A. Petosa. "An Overview of Tuning Techniques for Frequency-Agile Antennas". IEEE Antennas Propag. Mag. Vol. $54 \mathrm{~N}^{\circ}$ 5, pp. 271-296. October, 2012. ISSN: 1045-9243. DOI: $10.1109 /$ MAP.2012.6348178.

[2] F. Pizarro, R. Pascaud, O. Pascal, T. Callegari and L. Liard. "Evaluation of microplasma discharges as active components for reconfigurable antennas". In Proc. 6th Eur. Conf. Antennas Propag. (EuCAP), pp. 117-119. March, 2012.

[3] F. Pizarro, R. Pascaud, O. Pascal, T. Callegari and L. Liard. "Experimental study of RF/ microplasma interaction using an inverted microstrip line". In Proc. 7th Eur. Conf. Antennas Propag. (EuCAP), pp. 1187-1190. April, 2013. 
[4] T. Anderson. "Plasma Antennas". Artech House, 2011. ISBN-13: 978-1608071432.

[5] K.H. Becker, K.H. Schoenbach and J.G. Eden. "Microplasmas and applications". J. Phys. D Appl. Phys. Vol. 39 N $^{\circ} 3$, pp. R55. February, 2006. ISSN: 0022-3727. DOI: 10.1088/0022-3727/39/3/R01.

[6] P. Linardakis and G.G. Borg. "Small-Signal Impedance of a Radio Frequency Plasma Capacitor". IEEE Microw. Wireless Compon. Lett. Vol. $17 \mathrm{~N}^{\mathrm{o}} 11$, pp. 763-765. November, 2007. ISSN: 1531-1309. DOI: 10.1109/ LMWC.2007. 908042.
[7] P. Bhartia, I. Bahl, R. Garg and A. Ittipiboon. "Microstrip Antenna Design Handbook". Artech House. 2001. ISBN: 978-0890065136.

[8] R. Pascaud, F. Pizarro, O. Pascal, T. Callegari and L. Liard. "Theoretical and numerical study of a plasma-based frequency tunable microstrip antenna". In Proc. 8th Eur. Conf. Antennas Propag. (EuCAP), pp. 1545-154. April, 2014.

[9] S. Choudhury. "Effect of Dielectric Permittivity and Height on a Microstrip- Fed Rectangular Patch Antenna". International Journal of Electronics and Communication Technology (IJECT). Vol. 5, Issue Supplement 2, pp. 129130. January-March, 2014. 